\title{
Individual blood-brain barrier phenylalanine transport in siblings with classical phenylketonuria
}

\author{
J. Weglage ${ }^{1 *}$, D. WiedermanN ${ }^{2}$, J. DeneCKe $^{1}$, R. FeldmanN $^{1}$, \\ H.-G. KOCH ${ }^{1}$, K. UlLRICH ${ }^{3}$ and H. E. MÖLlER ${ }^{4,5}$ \\ ${ }^{1}$ Department of Pediatrics, University of Münster, Münster $;{ }^{2}$ Max-Planck-Institute \\ for Neurological Research, Cologne; ${ }^{3}$ Department of Pediatrics, University of \\ Hamburg, Hamburg; ${ }^{4}$ Institute for Physical Chemistry, University of Münster, \\ Münster; ${ }^{5}$ Institute for Clinical Radiology, University of Münster, Münster, \\ Germany
}

*Correspondence: Department of Pediatrics, University of Münster, Albert-Schweitzer-Str. 33,48129 Münster, Germany. E-mail:weglage@uni-muenster.de

MS received 15.02.02 Accepted 27.06.02

Summary: Recent studies indicate that individual blood-brain transport characteristics of phenylalanine may lead to different clinical outcomes in phenylketonuria (PKU) patients in spite of comparable dietary control. To check these preliminary data, we investigated four pairs of siblings with classical PKU (and identical genotype) using in vivo nuclear magnetic resonance spectroscopy in the course of an oral phenylalanine load ( $100 \mathrm{mg} / \mathrm{kg}$ body weight). Patients' brain phenylalanine concentrations were different in spite of similar blood levels. Interindividual variations of the apparent transport Michaelis constant, $K_{\mathrm{t}, \text { app }}$, ranged from 0.10 to $0.84 \mathrm{mmol} / \mathrm{L}$. The ratio of the maximal transport velocity, $T_{\max }$, over the intracerebral consumption rate, $V_{\text {met }}$, varied between 2.61 and 14.0. Siblings with lower values for $K_{\mathrm{t}, \text { app }}$, higher values for $T_{\max } / V_{\mathrm{met}}$, and higher concurrent brain phenylalanine levels showed a lower IQ and a higher degree of cerebral white matter abnormalities. The results indicate that blood-brain barrier transport characteristics and the resultant brain phenylalanine levels are causative factors for the individual clinical outcome in PKU.

Different clinical outcomes in spite of comparable dietary control are well known in patients with phenylketonuria (PKU; McKusick 261600), the most common treatable enzymopathy of amino acid metabolism in man (Scriver et al 2001). Recently we demonstrated that different blood-brain barrier (BBB) phenylalanine (Phe) transport characteristics do exist and that they correlate with clinical outcome in phenyl ketonuria (Möller et al 1998; Weglage et al 2001). For further evaluation we investigated data 
from in vivo nuclear magnetic resonance spectroscopy before and after an oral phenylalanine load (100 mg/ $\mathrm{kg}$ body weight) in four pairs of siblings with classical PKU. Siblings are most suitable for this investigation because of their identical genotype for PKU and their comparable socioeconomic background and dietary history.

\section{METHODS}

\section{Patients}

Four pairs of siblings with classical PKU (4 males and 4 females) ranged in age from 15 to 30 years (mean 20.4 years). Informed written consent was obtained in all cases after the nature and possible consequences of the study were fully explained. Consent to the study protocol was given by the local ethics committee.

Genotypes as well as extensive blood Phe testing (by high-performance liquid chromatography, HPLC) since birth indicated that all patients had classical PKU (see Table 1). None of them had any history of other factors disturbing brain development.

All patients started dietary treatment within the first two months of life (mean 20 days, range 12-37 days). They adhered to a Phe-restricted diet for different periods, at least up to the age of 10 years. Means of yearly medians of [Phe $]_{\text {blood }}$ ranged from 0.34 to $0.88 \mathrm{mmol} / \mathrm{L}$ for the first ten years of life $\left(\left\langle[\mathrm{Phe}]_{\text {blood }}\right\rangle_{<10 \mathrm{y}}\right)$ and from 0.74 to $1.42 \mathrm{mmol} / \mathrm{L}$ thereafter $\left(\left\langle[\text { Phe }]_{\text {blood }}\right\rangle \geqslant 10 \mathrm{y}\right)$.

\section{Assessment program}

Nuclear magnetic resonance spectroscopy: A $1.5 \mathrm{~T}$ whole-body magnetic resonance imager (Magnetom 63 SP, Siemens, Erlangen, Germany) and a standard head coil were used to record proton spectra from parieto-occipital white matter $(36 \mathrm{ml}$ volumes) employing the stimulated-echo technique (TR/TE 1600/20 ms, 512 acquisitions). Cerebral Phe concentrations were determined from difference spectroscopy versus spectra from healthy volunteers previously investigated (in 1998) as described in detail elsewhere (Möller et al 1998; Weglage et al 2001). In parallel with nuclear magnetic resonance (NMR) spectroscopy, $[\mathrm{Phe}]_{\text {blood, }}$, was measured quantitatively by HPLC.

To investigate BBB Phe-transport kinetics, time courses of [Phe $]_{\text {blood }}$ and $[\mathrm{Phe}]_{\text {brain }}$ were determined after an oral loading test with L-phenylalamine $(100 \mathrm{mg} / \mathrm{kg}$ body weight). After baseline measurement, $[\mathrm{Phe}]_{\text {blood }}$ and $[\mathrm{Phe}]_{\text {brain }}$ were recorded repeatedly starting $45 \mathrm{~min}$ after loading and extending from 24 to $36 \mathrm{~h}$. The patients had adhered to a fasting period of $12 \mathrm{~h}$ before loading (without amino acid supplement), and returned to a diet with $40-45 \mathrm{mg}$ Phe per kg body weight per day after the second post-load scan. The methods are described in detail elsewhere (Möller et al 1998; Weglage et al 2001).

Magnetic resonance imaging: Abnormalities of cerebral white matter were investigated by magnetic resonance imaging (MRI) using the same apparatus as for the spectroscopy investigations. 


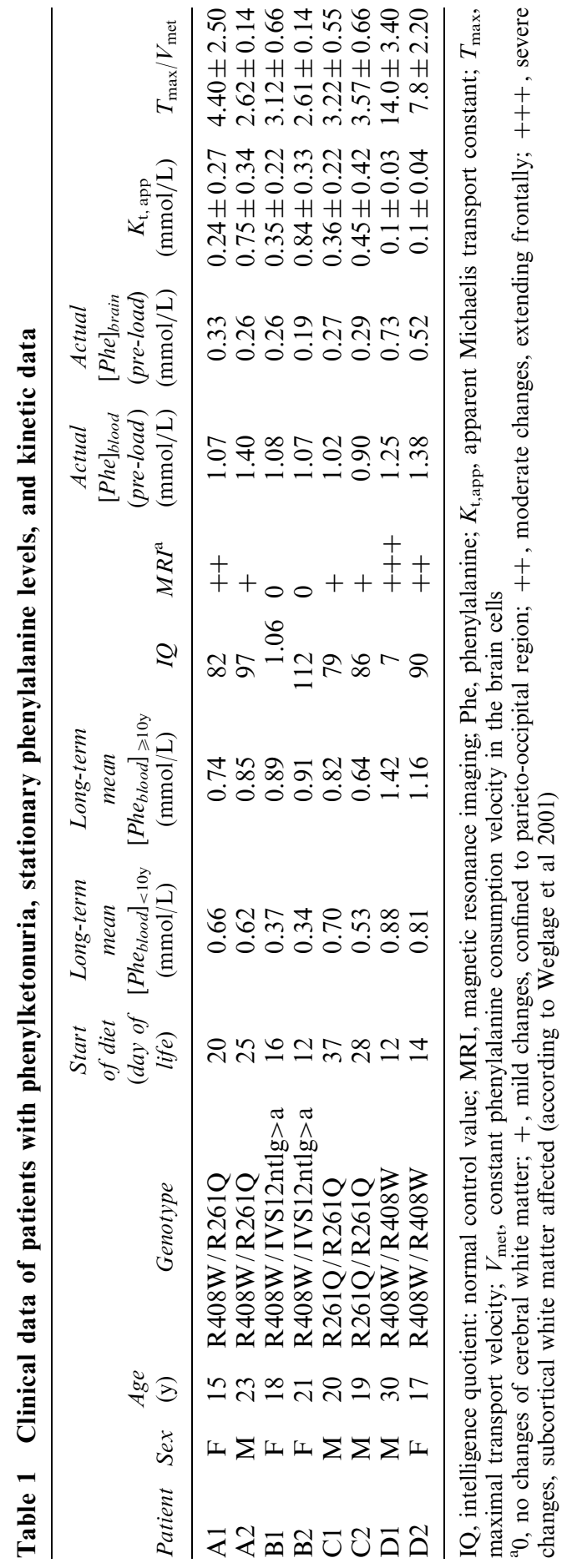


Intelligence testing: Intelligence quotient (IQ) was tested by the German version of the 'Culture Fair Intelligence Test - Scale 2' (CFT20) by Weiss. Patients reached a mean IQ within the normal range (mean 91; range 77-112).

Statistical analysis: Spearman rank correlations (SPSS for Windows, SPSS Inc., Chicago, IL, USA) were used to investigate the relation between spectroscopic parameters and results from MRI and intelligence testing. A significance level of $p=0.05$ was used throughout.

\section{RESULTS}

At blood Phe levels between 0.90 and $1.40 \mathrm{mmol} / \mathrm{L}$, concurrent [Phe] $]_{\text {brain }}$ ranged from 0.19 to $0.73 \mathrm{mmol} / \mathrm{L}$. Results of fitting the time courses are summarized for all patients in Table 1. In addition, example curves are shown for the siblings A1/A2 and B1/B2 in Figure 1. Generally, all parameters were distributed over fairly wide ranges: $K_{\mathrm{t} \text {,app }}$ varied between 0.10 and $0.84 \mathrm{mmol} / \mathrm{L}$, while interindividual variations of $T_{\max } / V_{\text {met }}$ ranged from 2.61 to 14.0. Siblings with lower values of $K_{\mathrm{t} \text {,app }}$ and higher ratios of $T_{\max } / V_{\text {met }}$, as well as higher preload [Phe] brain, showed more severe white matter abnormalities and lower IQs (Tables 1 and 2). Higher ratios of $T_{\max } / V_{\text {met }}$ are significantly correlated with pre-load $[\mathrm{Phe}]_{\text {brain }}$. In contrast, pre-load $[\mathrm{Phe}]_{\text {blood }}$ failed to reach significance.

\section{DISCUSSION}

As already discussed in previous publications, limitations of our technique lead to relatively large errors in the extracted [Phe] brain data (Möller et al 1995, 1998; Weglage et al 2001). However, current results fall within the range of data from our previous studies and a study from Koch and colleagues (2000). They further agree well with literature data obtained with a double indicator method (Knudsen et al 1995). In contrast to our findings, Rupp and colleagues (2001) reported a more constant relationship between brain and blood Phe concentrations. In addition, the number of pairs is small in this study.

However, the pre-load measurements indicate that interindividual differences in $[\mathrm{Phe}]_{\text {brain }}$ do exist even in siblings with identical genotypes of PKU. Such variations were significantly correlated with the degree of cerebral white matter abnormalities and with patient's intelligence scores. Three of the four siblings with comparable dietary history, higher IQs, and/or lower degrees of cerebral white matter changes had higher values for $K_{\mathrm{t} \text {,app }}$ and lower values for $T_{\max } / V_{\text {met }}$ and pre-load [Phe] $]_{\text {brain }}$. Pre-load [Phe $]_{\text {blood }}$ seems not to be of a prognostic value. Mild IQ differences in the siblings $\mathrm{C} 1$ and $\mathrm{C} 2$ may (partly) be explained by a significantly different quality of dietary control during the whole of life, especially during the first 8-10 years of life.

Owing to the observed trends (and being aware of the methodological shortcomings), we hypothesize that besides the quality of diet, favourable transport parameters (especially $K_{\mathrm{t}, \text { app }}$ ) may additionally protect the brain from uptake of high 

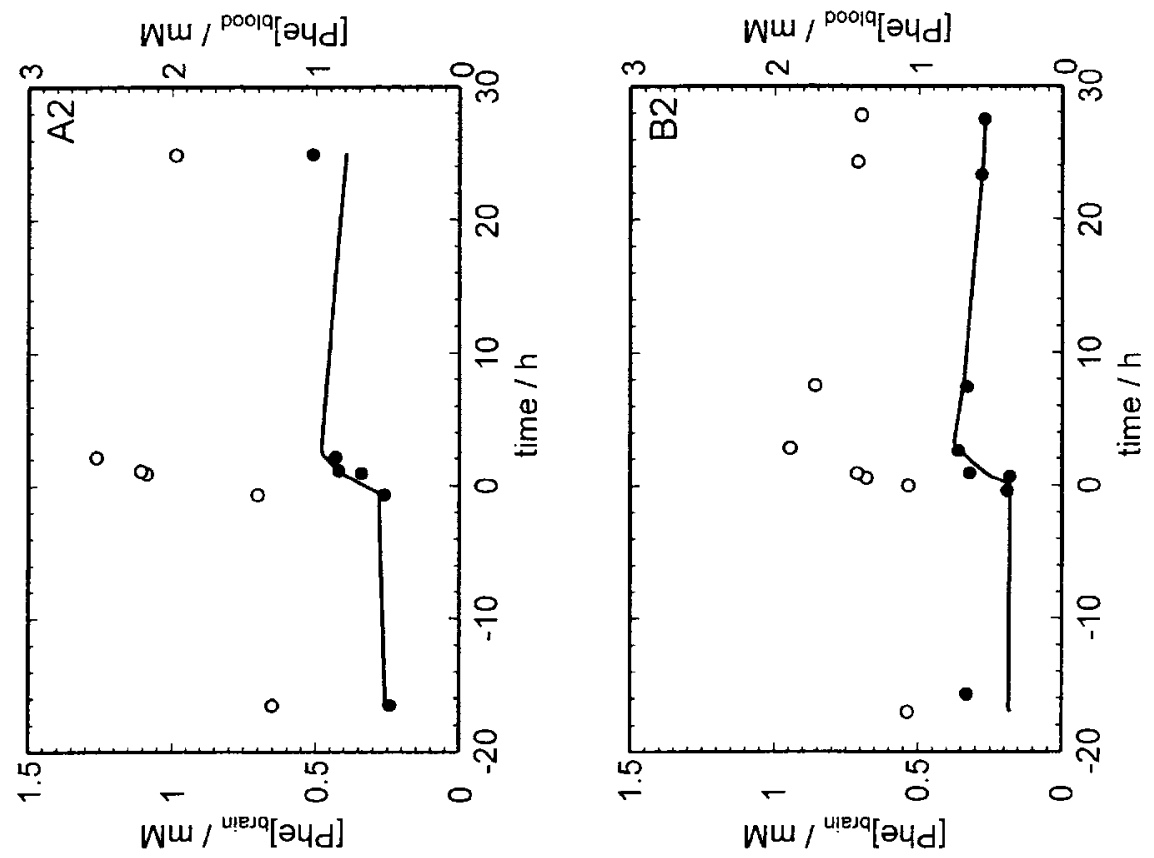

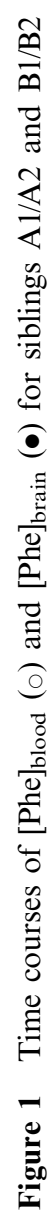
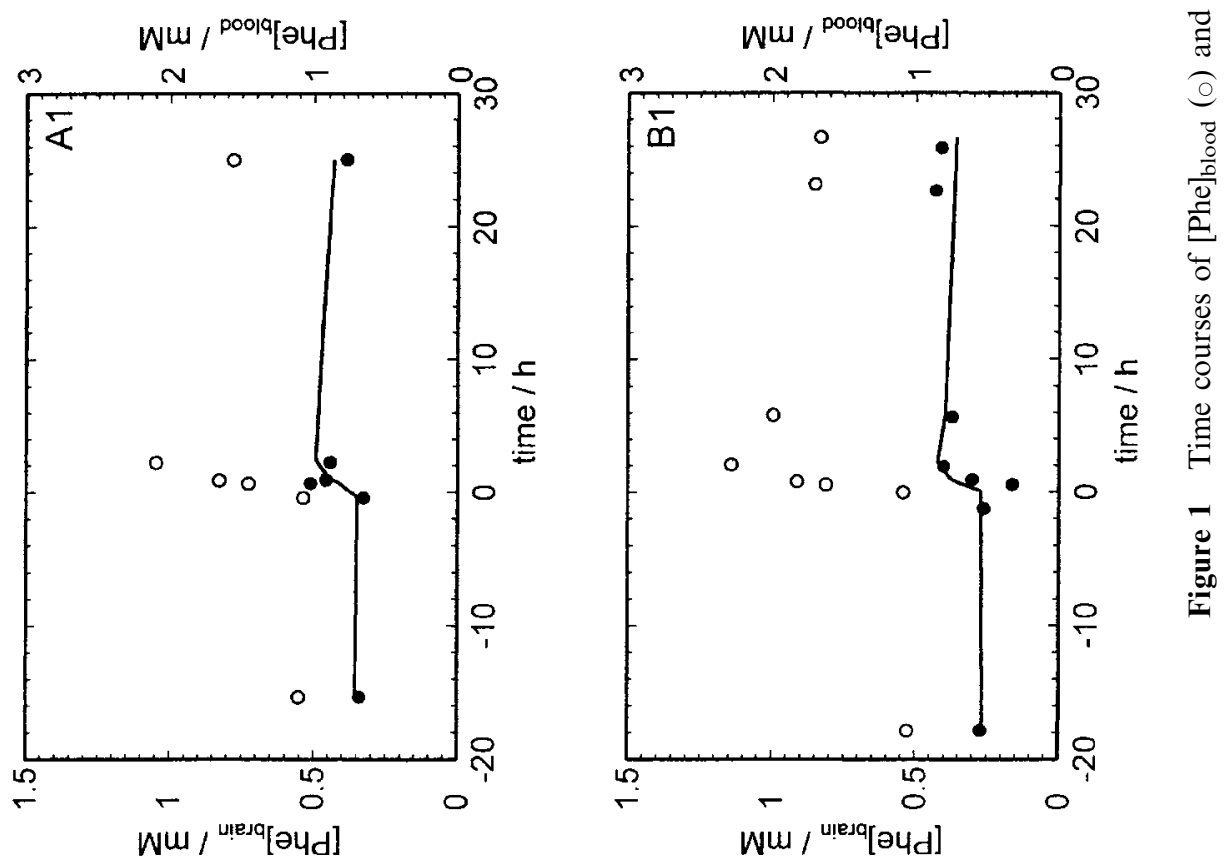

J. Inherit. Metab. Dis. 25 (2002) 
Table 2 Correlational findings

\begin{tabular}{|c|c|c|c|c|c|c|c|c|}
\hline & $I Q$ & $I Q p$ & $M R I$ & $M R I p$ & $K_{\mathrm{t}, \mathrm{app}}$ & $K_{\mathrm{t}, \mathrm{app}} p$ & $T_{\max } / V_{\mathrm{met}}$ & $T_{\max } / V_{\text {met }} p$ \\
\hline$K_{\mathrm{t}, \mathrm{app}}$ & 0.29 & NS & -0.70 & 0.05 & & & & \\
\hline$T_{\max } / V_{\text {met }}$ & -0.76 & 0.05 & 0.90 & 0.005 & & & & \\
\hline Pre-load [Phe $]_{\text {brain }}$ & -0.78 & 0.05 & 0.94 & 0.001 & -0.21 & NS & 0.98 & 0.0001 \\
\hline Pre-load [Phe $]_{\text {blood }}$ & 0.20 & NS & 0.27 & NS & 0.30 & NS & 0.39 & NS \\
\hline
\end{tabular}

Correlations according to Spearman two-tailed testing; NS, not significant

amounts of the neurotoxin Phe. More specifically, higher values of the Michaelis constant (i.e., a lower affinity of the transporter for Phe) and smaller $T_{\max } / V_{\text {met }}$ ratio lead to relatively lower intracerebral Phe concentrations at a given [Phe $]_{\text {blood }}$ value in siblings with identical gentotypes of PKU. Again, Phe blood-brain transport and $[\mathrm{Phe}]_{\text {brain }}$ seem to be important factors for the individual clinical outcome in PKU. However, further research is needed and the present results do not allow individual dietary recommendations. It is still unclear whether individual Phe transport characteristics are stable over time and what levels of cerebral Phe are safe in PKU.

\section{ACKNOWLEDGEMENT}

We thank all patients and their parents for participating in this study. Appreciation is extended to the SHS Company Heilbronn, Germany, for generous support.

\section{REFERENCES}

Knudsen GM, Hasselbalch S, Toft PB, Christensen E, Paulson OB, Lou H (1995) Blood-brain barrier transport of amino acids in healthy controls and patients with phenylketonuria. $J$ Inherit Metab Dis 18: 653-664.

Koch R, Moats R, Guttler F, Guldberg P, Nelson M (2000) Blood-brain phenylalanine relationships in persons with phenylketonuria. Pediatrics 106: 1093-1096.

Möller HE, Vermathen P, Ullrich K, Weglage J, Koch HG, Peters PE (1995) In-vivo NMR spectroscopy in patients with phenylketonuria: changes of cerebral phenylalanine levels under dietary treatment. Neuropediatrics 26: 199-202.

Möller HE, Weglage J, Wiedermann D, Ullrich K (1998) Blood-brain barrier phenylalanine transport and individual vulnerability in phenylketonuria. J Cereb Blood Flow Metab 18: 1184-1191.

Rupp A, Kreis R, Zschocke J, et al (2001) Variability of blood-brain ratios of phenylalanine in typical patients with phenylketonuria. J Cereb Blood Flow Metab 21: 276-284.

Scriver CH, Kaufman S, Eisensmith RC, Woo SLC (2001) The hyperphenylalaninemias. In Scriver CR, Beaudet AL, Sly WS, Valle D, eds; Childs B, Kinzler KW, Vogelstein B, assoc. eds. The Metabolic and Molecular Bases of Inherited Disease, 8th edn. New York: McGraw Hill, 1667-1724.

Weglage J, Wiedermann D, Denecke J, et al (2001) Individual blood-brain barrier phenylalanine transport determines clinical outcome in phenylketonuria. Ann Neurol 50: $463-467$. 